\title{
Narrow-Band Survey of Star Forming Regions
}

\author{
Movsessian T.A. ${ }^{* 1}$, Magakian T.Yu. ${ }^{\dagger 1}$, Dodonov S.N. ${ }^{\ddagger 2}$, and Andreasyan H.R. ${ }^{\S 1}$ \\ ${ }^{1}$ Byurakan Astrophysical Observatory, Aragatsotn reg., Byurakan, 0213 Armenia \\ ${ }^{2}$ Special Astrophysical Observatory, N.Arkhyz, Karachaevo-Cherkesia, 369167 Russia
}

\begin{abstract}
We present the results of a narrow-band $\mathrm{H} \alpha$ and [S II] imaging survey of the star-forming regions in Galaxy. Main tasks of this survey are the search and the studies of the new Herbig-Haro objects and collimated outflows, which are the main indicators of active processes of star formation. Besides, the eruptive stars of very rare types: FUors, EXors, UXors will be searched as well. As the main targets of this survey the $\mathrm{R}$ associations, young stellar objects associated with compact reflection nebulae as well as the deeply embedded infrared sources in molecular clouds are selected.

Observations are performed with 1-m Schmidt telescope at Byurakan Observatory, which is equipped with modern CCD detector. We plan to significantly expand the list of HH objects by using the high quantum efficiency of this system as well as the telescope's high focal ratio (F/2), which allows detecting low surface brightness objects, and its large field of view.
\end{abstract}

Keywords: Star formation, Herbig-Haro objects, jets and outflows

\section{Introduction}

For a long time Herbig-Haro objects $(\mathrm{HH})$ are recognised as a sign of high activity of star formation in molecular clouds. In fact, they represent shocked excitation zones where supersonic flows from young stellar objects (YSO) collide with interstellar medium and form small cloudlets with pure emission spectrum including permitted and low excitation forbidden emission lines ([O I $]$, $[\mathrm{S} \mathrm{II}]$ etc). Thus, the discovering of new $\mathrm{HH}$ objects is important as for the further studies of the phenomenon of directed outflows from young stars, as well as for the searches for new star forming regions and groups.

On the other hand, it is well known that the stellar sources of directed outflows, which have low and intermediate mass, often are associated with compact reflection nebulae of characteristic conical shape. Moreover, deep images in the optical and infrared ranges reveal their bipolar nature. Such shape is a consequence of the presence of circumstellar disks and conical cavities, created by matter outflow, near the YSOs illuminating these nebulae. In the overwhelming majority of cases HH objects and $\mathrm{HH}$ flows are located along the axes of these cometary nebulae, which proves a direct relationship between all these phenomena.

We continue our searches for $\mathrm{HH}$ objects in the dark clouds, started in Byurakan observatory more than twenty years ago, with the new equipment and wider-field telescope. Among the first targets we included in our program the Mon R1 and southern area of Mon R2 association (Racine, 1968), as well as some deeply embedded infrared sources. Here we present the first successful results.

\section{Observations}

The images were obtained on the nights of Feb. 3-4 2019 with the 1-m Schmidt telescope at Byurakan observatory, which was upgraded during $2013-2015$. The new detector is a reworked $4 \mathrm{~K} \times 4 \mathrm{~K}$

\footnotetext{
*tigmov@bao.sci.am, corresponding author

†tigmag@sci.am

${ }^{\ddagger}$ dodo@sao.ru

${ }^{\S}$ handreasyan@bao.sci.am
} 
Apogee (USA) liquid-cooled CCD camera with a pixel size of 0.868 arcsec and a field of view of about 1 square degree (Dodonov et al., 2017).

Narrow-band filters centered on $6560 \AA$ and $6760 \AA$, both with a full width at half-maximum (FWHM) of $100 \AA$, were used to obtain $\mathrm{H} \alpha$ and [S II] images, respectively. A mid-band filter, centered on $7500 \AA$ with a FWHM of $250 \AA$, was used for the continuum imaging.

A dithered set of 5 min exposures was obtained in each filter. The effective exposure time in $\mathrm{H} \alpha$ equaled $6000 \mathrm{sec}$, in [S II] - $7200 \mathrm{sec}$ and in the continuum - $2400 \mathrm{sec}$. Images were reduced in the standard manner using IDL package developed by one of authors (SND), which includes bias subtraction, cosmic ray removal, and flat fielding using "superflat field", constructed by several images.

\section{Results}

\subsection{Mon R1}

The area under study included three bright reflection nebulae (NGC 2245, NGC 2247 and IC 446), as well as several isolated dark lanes and emission-reflection filaments, which are stretched in SE-NW direction through the whole field. Mon R1 contains at least 30 YSOs, which were discovered in the optical range (Herbst et al., 1982). The distance of Mon R1 is usually assumed to be 800 pc, as is that of the nearby Mon OB1 (see Dahm (2003), for a detailed review). The YSOs in Mon R1 can be spatially divided into two groups: one group includes NGC 2245, 2247 and IC 446, and the other is projected on the large reflection nebula IC 2169. Recent infrared and radio observations indicate significant star-forming activity in the latter group (Bhadari et al., 2020). In comparison to the adjacent Mon OB1, Mon R1 has received much less attention. Nearly all observational studies were concentrated on IC 446, where a compact group of emission-line stars, including the HAeBe star VY Mon, was found by Cohen \& Kuhi (1979). No searches for HH objects in the Mon R1 field have been performed to date.

Our observations immediately revealed a considerable amount of totally new HH knots and groups, standing out by their pure emission-line spectrum, in this area (Movsessian et al., 2021, in press). Besides, in the course of HH knots search, we noted several nebulous stars, which can be the probable members of Mon R1. Some of them can represent exciting sources of HH objects.

A $\mathrm{H} \alpha+\left[\mathrm{S} \mathrm{II}_{\mathrm{II}}\right]$ image, which is covering one square degree field of the Mon R1 region, is shown on Fig.1. The zones with newly discovered $\mathrm{HH}$ objects and $\mathrm{HH}$ flows are marked by rectangles. Full results are presented in the forthcoming paper (Movsessian et al., 2021, in press). Here we show two most interesting outflow systems.

\subsubsection{HH 1196 flow}

This chain of $\mathrm{HH}$ objects contains at least six distinct knots with diffuse emission between them; its total length is about 4 arcmin (Fig. 2). All knots are aligned along an axis with position angle of 155. Knots A, B, E and F have bow-shape morphology with apexes pointing in S-SE direction. This fact confirms the impression of a single collimated outflow from a source, located in the northern direction.

As can be seen from the figure, the relative intensities of the individual knots in $\mathrm{H} \alpha$ and $[\mathrm{S} \mathrm{II}]$ vary. Knots $\mathrm{A}, \mathrm{B}, \mathrm{C}$ and $\mathrm{D}$ are relatively brighter in $\mathrm{H} \alpha$, while knot $\mathrm{E}$ has the ratio of $\mathrm{H} \alpha$ and $[\mathrm{S}$ II] near 1 , and knot $\mathrm{F}$ is seen mainly in $[\mathrm{S} \mathrm{II}]$.

Along the line connecting the HH 1196 knots, several objects, any of which can be suspected as a possible source of this outflow, can be found.

Total length of this HH outflow is about $1.6 \mathrm{pc}$; thus, it represents so-called giant HH outflow.

\subsubsection{HH 1203}

This HH flow includes four HH objects in a chain, embedded in the general diffuse emission (marked in Fig. 3 as knots A, B, C and D). Brightest knot (B) in this chain is somewhat displaced from the axis of whole complex. To the south-east from this HH group there is a compact cone-shaped reflection 


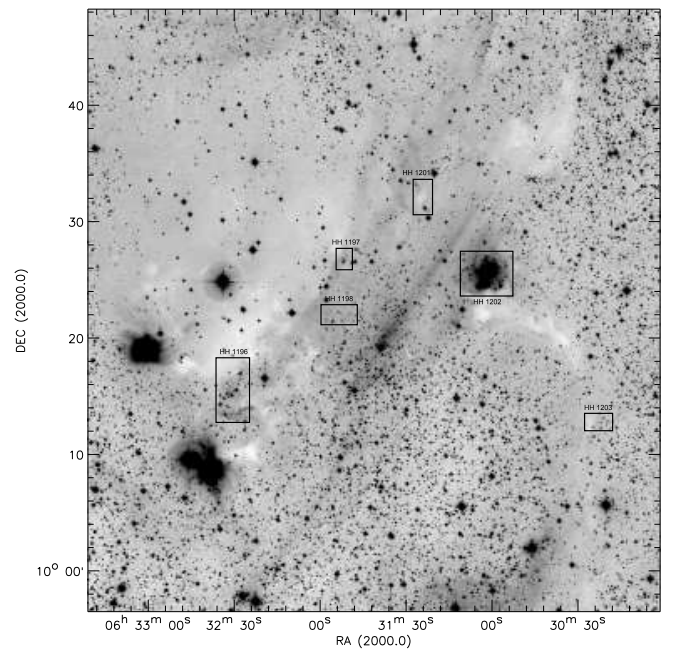

Figure 1. Whole observed field in $\mathrm{H} \alpha+[\mathrm{S} \mathrm{II}]$ emission, built from the images, obtained with $1 \mathrm{~m}$ telescope. The areas including newly discovered HH objects and outflows, are shown by rectangles. Also three major reflection nebulae are marked.
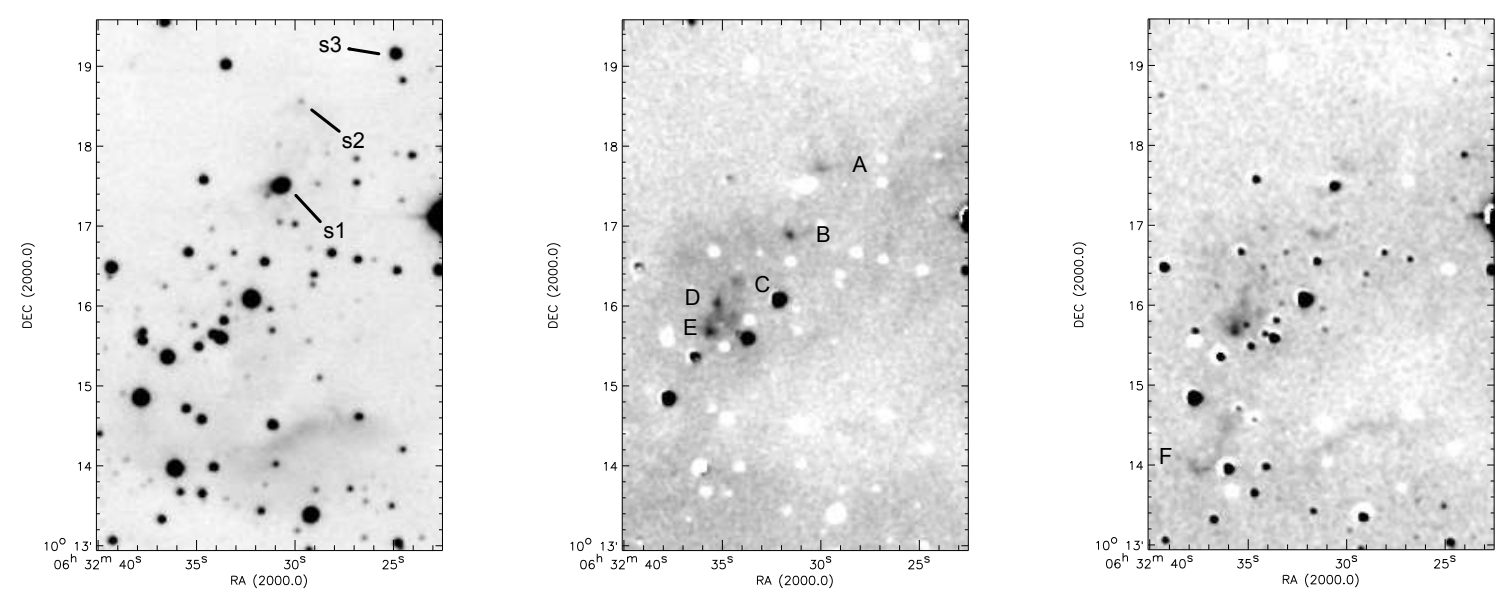

Figure 2. Images of HH 1196 outflow system: continuum image (left panel), continuum subtracted $\mathrm{H} \alpha$ (central panel) and continuum subtracted [S II] (right panel). By s1, s2 and s3 the 2MASS J06323159+1017352, IRAS 06297+1021 (E) and IRAS 06297+1021 (W) objects are marked respectively on the left panel. 

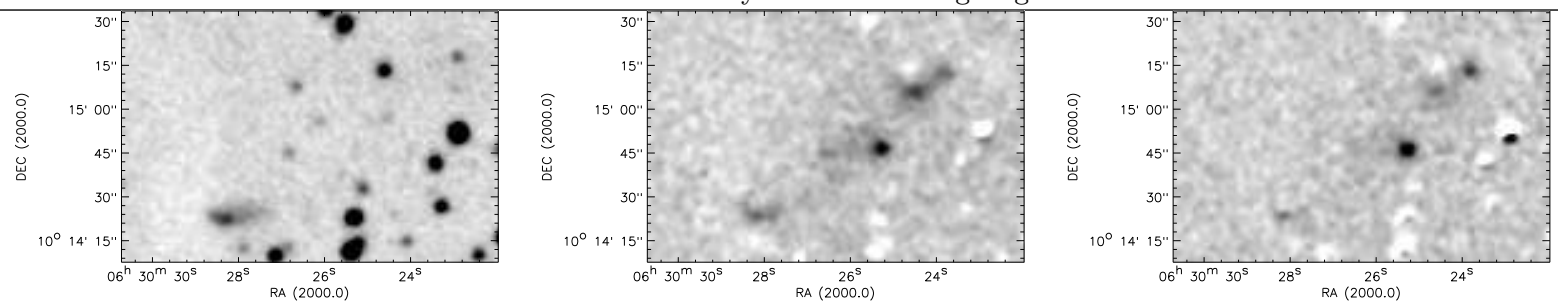

Figure 3. Images of HH 1203 outflow system: continuum image (left panel), continuum subtracted $\mathrm{H} \alpha$ (central panel) and continuum subtracted [S II] (right panel).

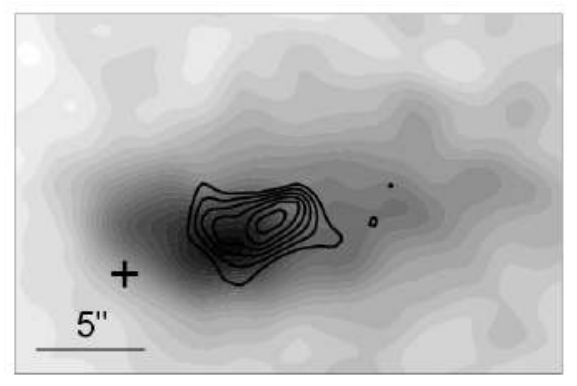

Figure 4. Image of the reflection nebula near HH 1203 in continuum (gray scale) and in [S II] emission (isolines). The position of IRAS $06277+1016$ infrared source is marked by the cross. Short emission jet, pointing in the north-west direction along the axis of $\mathrm{HH}$ flow, is well seen.

nebula, which lies near the axis of this elongated group. On Fig. 4 the continuum image of this reflection nebula with $[\mathrm{S} \mathrm{II}]$ image overlaid is presented. This combination reveals the short emission jet, elongated in the direction of the axis of HH 1203 flow. Its existence confirms the assumption that the star, obviously embedded into the nebula, and seen as an infrared source IRAS $06277+1016$, also is the source of HH 1203.

\subsection{Mon R2-south}

Mon R2 is a well studied region of star formation which contains early-type stars (Racine, 1968), molecular outflows (Meyers-Rice \& Lada, 1991), an embedded HII region (Downes et al., 1975) and clusters of infrared sources (Thronson et al., 1980). However, the amount of known before HH objects in this field is low; they were found mainly on the eastern side of Mon R2 (Carballo \& Eiroa, 1993). The distance of Mon R2 is estimated as $\approx 900$ pc (Lombardi et al., 2011). Our attention was drawn to the area to the south from the central part of Mon R2, where several nebulous objects and an eruptive star V899 Mon are located.

A $\mathrm{H} \alpha+[\mathrm{S} \mathrm{II}]$ image, which is covering one square degree field of the Mon R2 region, is shown on Fig.5. The zones with newly discovered HH objects and HH flows are marked by rectangles.

\subsubsection{V899 Mon}

A possible FUor-type eruption of V899 Mon(IRAS 06068-0641), the star, located near the Monoceros R2 region, was first discovered during the Catalina Real-time Transient Survey (CRTS) and reported by Wils et al. (2009). They announced the source as a FUor candidate, which suggestion was based on the constant brightening it has been undergoing since 2005. The spectrum published by Wils et al. (2009) showed strong $\mathrm{H} \alpha$ and CaII IR triplet lines, which identify the outbursting source as a YSO.

Further optical and near-infrared spectroscopy of V899 Mon confirmed it to be a member of the FUors/EXors family of outbursts. Photometrically and spectroscopically V899 Mon's properties lie between EXors and classical FUors (Ninan et al., 2015). But it is probably more similar to EXors than to the classical FUors. 


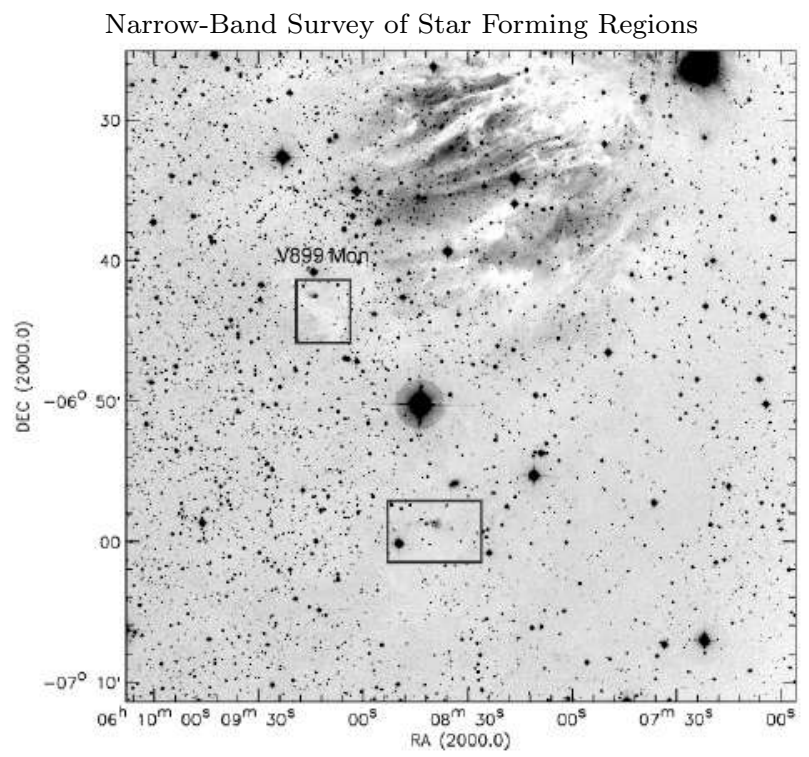

Figure 5. Whole observed field of Mon R2 south in $\mathrm{H} \alpha+[\mathrm{S} \mathrm{II}]$ emission, built from the images, obtained with $1 \mathrm{~m}$ telescope. The areas including newly discovered $\mathrm{HH}$ objects and outflows, are shown by rectangles.
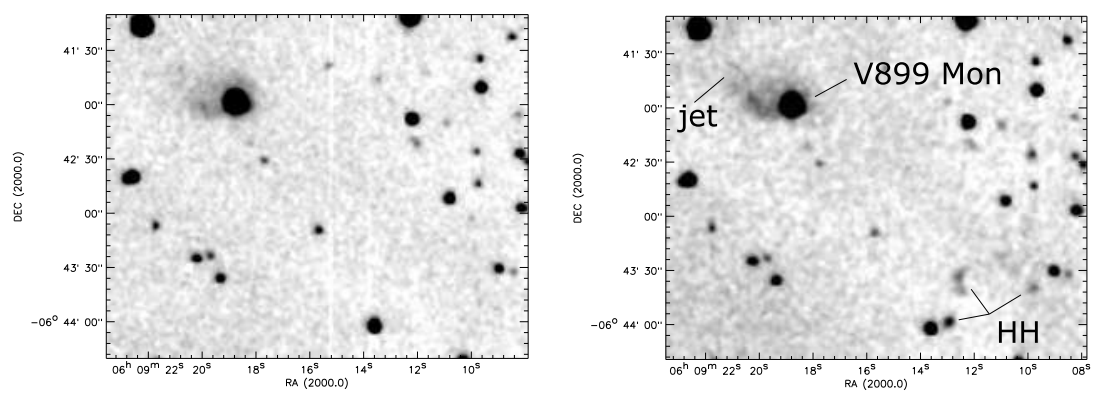

Figure 6. Images of V899 Mon and its outflow system: continuum image (left panel) and [S II] image (right panel).

On the distance of about 2.5 arcmin in south-west from V899 Mon we discovered four HH knots (Fig.6); besides, the narrow emission-line jet, oriented along the axis of the reflection nebula, associated with V899 Mon, was discovered (Fig.4). These data suggest that V899 Mon, like several other FU Ori type objects, is also a source of the collimated, possibly bipolar outflow, because the counter flow can be represented by newly found HH knots. However, one cannot exclude that these HH objects could be produced by other infrared sources in this area.

\subsubsection{Curved HH flow}

About 20 arcmin towards south-west from V899 Mon we revealed several HH knots near 2MASS 06084223-0657385 infrared source, which is associated with bipolar reflection nebula. HH objects lie on a parabolic curve at the apex of which this infrared source is located (Fig.7). It is remarkable that near the source this line coincides with the axis of the above mentioned bipolar nebula. Therefore, it can be argued that we are dealing with the bipolar collimated outflow of an unusual arc-shaped structure. Such a morphology is typical as for so-named irradiated jets as for outflows from the sources with high proper motion. We incline to choose the second scenario, because irradiated outflows usually are represented by very thin emission filaments without prominent internal knotty structures (Bally \& Reipurth, 2001). Taking this scenario into account we estimated the value of the proper motion of the source, which turned to be about $50 \mathrm{~km} \mathrm{~s}^{-1}$, which is quite reasonable. Estimated total length of this bipolar outflow for the distance of $900 \mathrm{pc}$ will be about 1.5 pc. Thereby, this outflow system as well as HH 1196 represents giant or so called parsec-scale HH flow. 

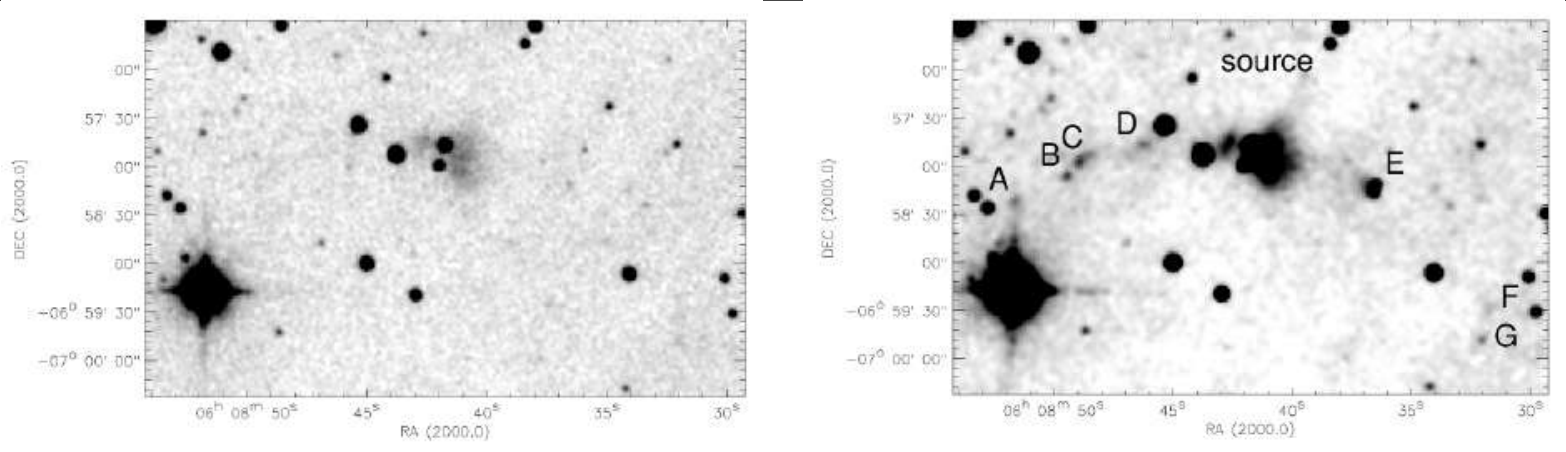

Figure 7. Images of curved HH outflow system: continuum image (left panel) and [S II] $+\mathrm{H} \alpha$ image (right panel).
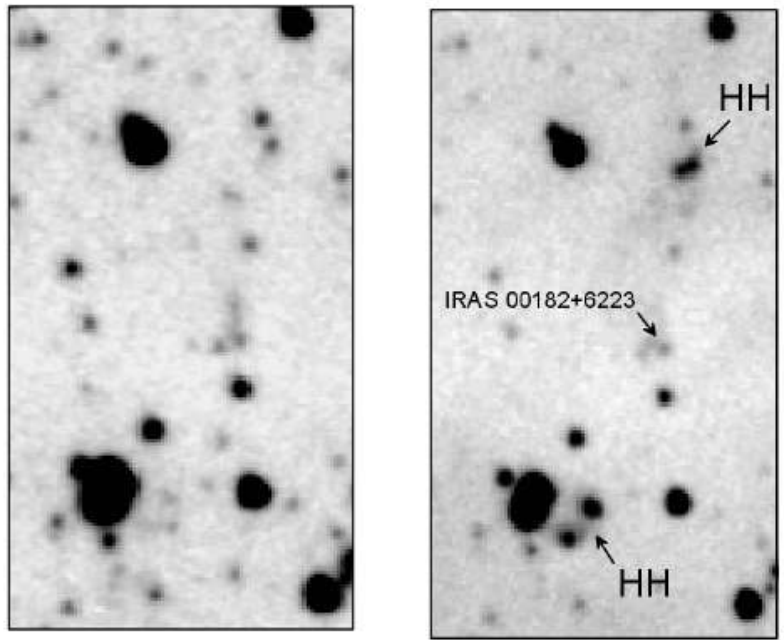

Figure 8. Images of IRAS 00182+6223 outflow system: continuum image (left panel), H $\alpha$ image (right panel).

\subsection{IRAS $00182+6223$}

This poorly investigated object, located at distance of $4.68 \mathrm{kpc}$ firstly was mentioned as a source of CO outflow (Wouterloot \& Brand, 1989). Observations in near-infrared range revealed small reflection nebula extending to the north from IRAS 00182+6223 (Connelley, Reipurth, \& Tokunaga, 2007).

Our observations revealed two emission lobes in northern and southern sides of IRAS $00182+6223$ with total length of about 1.7 arcmin $(2.3 \mathrm{pc}$ for the distance of $4.68 \mathrm{kpc}$ ) (Fig.8). It is worth to mention that this source is located in the dark cloud, which is covered by foreground stars of Milky Way. It would be one of the brightest known $\mathrm{HH}$ objects, if is was located at a distance of Orion star forming region.

\section{Conclusion}

Since the beginning of 2019, when the survey began, more than 30 new HH objects and outflow systems have been found. Among them several giant outflow systems, narrow jets as well as curved HH flow should be mentioned.

In addition to all the results presented above, this work demonstrates that the 1-m Schmidt telescope of Byurakan Observatory, which was used several decades ago for well-known surveys of active galaxies such as the First Byurakan Survey and Second Byurakan Survey, can still lead to important discoveries. 


\section{Acknowledgements}

This work is based on observations conducted with the 1-m Schmidt telescope of the Byurakan Astrophysical Observatory of the National Academy of Sciences of Armenia. This work was supported by the Republic of Armenia State Committee of Science, in the framework of research project number $18 \mathrm{~T}-1 \mathrm{C}-329$.

\section{References}

Bally J., Reipurth B., 2001, ApJ, 546, 299.

Bhadari N. K., Dewangan L. K., Pirogov L. E., Ojha D. K., 2020, ApJ, 899, 167.

Carballo R., Eiroa C., 1993, in Stellar Jets and Bipolar Outflows, Proceedings of the 6th International Workshop, Ap\&SpSc Lib. 186, p.213

Cohen M., Kuhi L.V., 1979, ApJS, 41, 743

Connelley M. S., Reipurth B., Tokunaga A. T., 2007, AJ, 133, 1528.

Dahm S.E, 2008, in Reipurth B., ed., Handbook of Star Forming Regions, Vol. I, Astron. Soc. Pac., p. 966

Dodonov S.N., Kotov S.S., Movsesyan T.A., Gevorkyan M. 2017, Astrophysical Bulletin, 72, 473

Downes D., Winnberg A., Goss W. M., Johansson L. E. B., 1975, A\&A, 44, 243

Herbst W., Miller D.P., Warner J.W., Herzog A., 1982, AJ, 87, 98

Lombardi, M., Alves, J., Lada, C.J., 2011, A\&A, 535, A16

Meyers-Rice B. A., Lada C. J., 1991, ApJ, 368, 445.

Movsessian T.A., Magakian T.Yu., Dodonov S.N., 2021, MNRAS, 500, 2440

Ninan, J.P., Ojha, D.K., Baug, T., Bhatt, B.C., Mohan, V., Ghosh, S.K., Men'shchikov, A., Anupama, G.C., Tamura, M., Henning, Th., 2015, ApJ, 815, 4

Racine R., 1968, AJ, 73, 233

Thronson H. A., Gatley I., Harvey P. M., Sellgren K., Werner M. W., 1980, ApJ, 237, 66.

Wils P., Greaves J., Drake A. J., Catelan M., 2009, Central Bureau Electronic Telegrams, 2033, 1

Wouterloot J. G. A., Brand J., 1989, A\&AS, 80, 149 